\section{(6) OPEN ACCESS}

\title{
The Thr300Ala variant in ATG16L1 is associated with improved survival in human colorectal cancer and enhanced production of type I interferon
}

\author{
Wesley A Grimm, ${ }^{1}$ Jeannette S Messer, ${ }^{1}$ Stephen F Murphy, ${ }_{1}^{1}$ Thomas Nero, ${ }^{1}$ \\ James P Lodolce, ${ }^{1}$ Christopher R Weber, ${ }^{2}$ Mark F Logsdon, ${ }^{1}$ Sarah Bartulis, ${ }^{1}$ \\ Brooke E Sylvester, ${ }^{1}$ Amanda Springer, ${ }^{1}$ Urszula Dougherty, ${ }^{1}$ Timothy B Niewold, ${ }^{1}$ \\ Sonia S Kupfer, ${ }^{1}$ Nathan Ellis, ${ }^{1}$ Dezheng Huo, ${ }^{1}$ Marc Bissonnette, ${ }^{1}$ David L Boone ${ }^{3}$
}

- Additional material is published online only. To view please visit the journal online (http://dx.doi.org/10.1136/ gutjnl-2014-308735).

${ }^{1}$ Departments of Medicine, The University of Chicago, Chicago, Illinois, USA ${ }^{2}$ Departments of Pathology, University of Chicago, Chicago, Illinois, USA

${ }^{3}$ Departments of Microbiology and Immunology, Indiana University School of Medicine - South Bend, South Bend, Indiana, USA

\section{Correspondence to}

Dr David Boone, Departments of Microbiology and Immunology, Indiana University School of Medicine, 1234 N. Notre Dame Ave., South Bend, IN 46617, USA; daboone@iu.edu

Received 29 October 2014 Revised 8 December 2014 Accepted 11 December 2014 Published Online First 2 February 2015

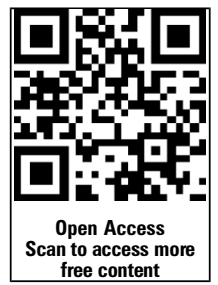

CrossMark

To cite: Grimm WA, Messer JS, Murphy SF, et al. Gut 2016;65:456-464.

\section{ABSTRACT}

Objective ATG16L1 is an autophagy gene known to control host immune responses to viruses and bacteria. Recently, a non-synonymous single-nucleotide polymorphism in ATG16L1 (Thr300Ala), previously identified as a risk factor in Crohn's disease (CD), was associated with more favourable clinical outcomes in thyroid cancer. Mechanisms underlying this observation have not been proposed, nor is it clear whether an association between Thr300Ala and clinical outcomes will be observed in other cancers. We hypothesised that Thr300Ala influences clinical outcome in human colorectal cancer (CRC) and controls innate antiviral pathways in colon cancer cells.

Design We genotyped 460 patients with CRC and assessed for an association between ATG16L1 Thr300Ala and overall survival and clinical stage. Human CRC cell lines were targeted by homologous recombination to examine the functional consequence of loss of ATG16L1, or introduction of the Thr300Ala variant.

Results We found an association between longer overall survival, reduced metastasis and the ATG16L1 Ala/Ala genotype. Tumour sections from ATG16L1 Ala/ Ala patients expressed elevated type I interferons (IFN-I)inducible, MxA, suggesting that differences in cytokine production may influence disease progression. When introduced into human CRC cells by homologous recombination, the Thr300Ala variant did not affect bulk autophagy, but increased basal production of type I IFN. Introduction of Thr300Ala resulted in increased sensitivity to the dsRNA mimic poly(l:C) through a mitochondrial antiviral signalling (MAVS)-dependent pathway.

Conclusions The CD-risk allele, Thr300Ala, in ATG16L1 is associated with improved overall survival in human $C R C$, generating a rationale to genotype ATG16L1 Thr300Ala in patients with CRC. We found that Thr300A alters production of MAVS-dependent type I IFN in CRC cells, providing a mechanism that may influence clinical outcomes.

\section{INTRODUCTION}

ATG16L1 forms a complex with ATG5 and ATG12 to mediate conjugation of LC3 to the developing autophagophore, a process essential for

\section{Significance of this study}

What is already known on this subject?

- ATG16L1 is a core autophagy protein.

- A genetic non-synonymous polymorphism in ATG16L1 causing an amino acid change T300A is associated with risk for Crohn's disease (CD).

- ATG16L1 also has functions not related to autophagy.

\section{What are the new findings?}

- Patients with colorectal cancer who have the CD-associated ATG16L1 T300A variant have better long-term survival than patients who have the T300-associated genotype.

- Human cells genetically engineered to have the T300A mutation produce more type I interferon than parental cells without the mutation.

- The T300A mutation results in higher type I interferon production by a mitochondrial antiviral signalling (MAVS)-mediated signalling pathway.

\section{How might it impact on clinical practice in} the foreseeable future?

- Patients with colorectal cancer with T300A mutation may derive more benefit from adjuvant-based therapies targeting the mitochondrial MAVS antiviral pathway. 
more favourable clinical outcomes in patients with thyroid carcinoma. ${ }^{10}$ Whether this association is specific to thyroid cancer or can be found in other malignancies is unknown. Furthermore, potential functions of T300A in cancer cells that may account for this observation have not been proposed.

Type I interferons (IFNs) are essential for the initiation of antiviral immunity, but also participate in antitumour host responses. ${ }^{11}$ Type I IFN inhibits cancer cell growth, ${ }^{12}{ }^{13}$ reduces metastasis ${ }^{14-16}$ and increases antitumor host immunity. ${ }^{17-20}$ Type I IFN-I are a class of $\alpha$-helical cytokines represented by several semi-homologous IFN- $\alpha$ genes and a single IFN- $\beta$. IFN- $\alpha / \beta$ are produced primarily in response to cytosolic viral dsRNA and 5'triphosphate ssRNA, but also dsDNA and lipopolysaccharide in some cell types. ${ }^{11}$ Toll-like receptor 3 (TLR3) and mitochondrial antiviral signalling protein (MAVS) pathways are the primary routes leading to phosphorylation of interferon regulatory factors (IRFs, including IRF5, IRF7 and IRF3) and downstream transcription of type I IFNs. ${ }^{11}$ Activation of MAVS occurs when the cytosolic helicases retinoic acid inducible gene I (RIG-I) and melanoma differentiation-associated protein 5 (MDA5) bind to viral dsRNAs, leading to interaction of homotypic caspase recruitment domain (CARD domain) and oligomerisation of MAVS on the surface of the mitochondria. ${ }^{21-23}$

Given the known role of ATG16L1 in regulation of host immune responses in the GI tract, we hypothesised that the T300A variant is associated with altered clinical outcomes in colorectal cancer (CRC). To test this, we examined 460 patients treated at the University of Chicago and identified a beneficial effect of ATG16L1 Ala/Ala genotype on overall survival. We found that fewer metastatic cancers are diagnosed among patients carrying the ATG16L1 Ala/Ala genotype, and that tumour sections from ATG16L1 Ala/Ala patients express elevated IFN-I-inducible genes. When introduced into human CRC cells by homologous recombination, T300A resulted in increased production of IFN-I. Cultured CRC cells expressing T300A displayed increased sensitivity to the dsRNA mimic poly(I:C) through a MAVS-dependent pathway. Thus, ATG16L1 T300A is associated with increased overall survival in human CRC patients, reduced metastasis and elevated MAVS-dependent IFN-I in human CRC cells. These results may have applications regarding the use of oncolytic viruses and $\mathrm{p}(\mathrm{I}: \mathrm{C})$ as an immunoadjuvants to cancer therapy.

\section{MATERIALS AND METHODS}

Please refer to the online supplementary methods section for a description of patient selection criteria, statistical analysis, and other methods and reagents that could not be outlined here due to space constraints.

\section{Isolation of mitochondrial membranes}

Briefly, cells were washed twice in ice-cold phosphate-buffered saline, collected in sucrose buffer $(320 \mathrm{mM}$ sucrose, $1 \mathrm{mM}$ EDTA, $10 \mathrm{mM}$ Tris-HCl ( $\mathrm{pH}$ 7.4)), and subject to $10-15$ strokes using a Dounce-type homogeniser. Nuclei were cleared twice from homogenates by centrifugation at $3300 \mathrm{rpm}$ at $4^{\circ} \mathrm{C}$, and the mitochondrial membrane fraction was pelleted by a single centrifugation at $12000 \mathrm{rpm}\left(4^{\circ} \mathrm{C}\right.$, $20 \mathrm{~min})$. Cytosolic fractions were collected and membrane pellets washed in sucrose buffer once before lysis in radioimmunoprecipitation assay buffer, protein quantification and immunoblotting.

\section{IFN-induced GTP-binding protein Mx1 (MxA) immunohistochemisty scoring}

Patient tissues were obtained from the Human Tissue Resource Center at the University of Chicago. Tissues examined were derived from surgically resected tumours from patients diagnosed with invasive colon cancer. These slides were processed and stained for MxA as described above. MxA scoring was performed by a board-certified, trained pathologist who was blinded to patient genotypes and other clinical data. Slides were scored for MxA expression within cancer epithelium on a scale of $1-4$ related to the extent of MxA positivity. $1=<5 \%$ positive, $2=<10 \%$ positive, $3=<30 \%$ and $4=>30 \%$ positive. When biological replicates (two tumours per patient) were available, both tumours were stained for MxA protein and scores were averaged to produce a single value per patient. Values were plotted and statistical significance was determined using Fisher's exact test using STATA.

\section{WISH cell stimulation}

WISH cells are a HeLa cell line (ATCC, CCL-25) highly sensitive to stimulation with IFN-I and are used to assay IFN-I activity as previously described. ${ }^{24}$ Human recombinant IFN- $\beta$ is used as a positive control. Briefly, conditioned media was collected from cells cultured in 6-well plates after reaching $75 \%$ confluence. Afterwards cells were dissociated and quantitated using a haemocytometer for normalisation. Prior to stimulation, $0.5 \times 10^{6}$ WISH cells were seeded in 6 -well plates. On the following day, conditioned media was used to stimulate WISH cells for $4 \mathrm{~h}$. After stimulation, media was aspirated and cells were immediately lysed using Trizol reagent (Invitrogen). RNA was isolated according to manufacturer's instructions and used for subsequent cDNA synthesis. Real-time PCR was performed to evaluate induction of IFN-I sensitive genes.

\section{RESULTS}

\section{Patient demographics}

In a cohort of 460 patients diagnosed with invasive CRC at the University of Chicago Medical Center between 1992 and 2012, $56 \% \quad(\mathrm{n}=258) \quad$ were Caucasian and $44 \% \quad(\mathrm{n}=202)$ African-American. Mean age of diagnosis was 64.5 years (range 21-93) and was greater in African-Americans compared with Caucasians (67.4 vs 62.1, one-way analysis of variance $\mathrm{p}<0.001)$. Allelic frequencies at rs2241880 did not differ significantly from those found in public databases available (dbSNP) (see online supplementary table S1). In agreement with published data (National Center for Biotechnology Information dbSNP), genotypic frequencies were significantly different when comparing African-American and Caucasian individuals with CRC, the Ala/Ala genotype (GG) being less prevalent in the former $(13 \%$ vs $28 \%, \mathrm{p}<0.001$, see online supplementary table S2). Comparing the clinicopathology of disease in Caucasians and African-Americans revealed fewer rectal cancers diagnosed in African-Americans $(\mathrm{p}<0.001)$; however, there were no differences in tumour stage or grade at diagnosis, receipt of radiation and/or chemotherapy, or histopathological features of disease with respect to race (see online supplementary table S2).

\section{Association of ATG16L1 T300A with prolonged overall survival}

Mean age of diagnosis did not differ significantly by ATG16L1 genotype (see online supplementary table S2). Overall mean follow-up was 40.7 months (range, 0.98-173) and did not differ by race $(p=0.76)$ (see online supplementary table S2). However, 
follow-up time was significantly increased in Ala/Ala patients with colon cancer $(p=0.048)$ (see online supplementary table S3) but not rectal cancer. Over the period of observation, there were 129 deaths from all causes. Disease-specific mortality data were not available for this cohort. The Kaplan-Meier method was used to estimate of survival probability with respect to ATG16L1 genotype while adjusting for race and location of the primary tumour (see online supplementary figure S1). Improved overall survival of patients with CRC was observed in Ala/Ala individuals, which reached statistical significance when plotted in a recessive model $(p=0.044$, figure 1$)$. These results suggest that estimated survival is improved in patients carrying the Ala/Ala genotype (GG) in ATG16L1.

Prognostic factors were evaluated by univariate Cox regression. This method identified a survival benefit in patients with CRC carrying the ATG16L1 Ala/Ala genotype (GG) $(\mathrm{HR}=0.55$, $\mathrm{p}=0.017$ ) in addition to other prognostic factors, including age, race, stage, grade and location of the tumour (see online supplementary table S4). Multivariate analysis indicated that ATG16L1 genotype was no longer a significant prognostic factor with respect to overall survival $(\mathrm{HR}=0.73, \mathrm{p}=0.22)$ while race, invasive stage and grade remained significant. These data suggest that the T300A variant may not act as an independent factor with respect to overall survival and may be associated with a tumour characteristic included in the multifactorial model.

\section{Association of ATG16L1 T300A with fewer stage IV colon cancers}

In order to understand the effect underlying an association between ATG16L1 T300A and improved overall survival, we tested for relationships between ATG16L1 genotype and clinicopathology of disease. We did not detect associations between ATG16L1 genotype and radiation/chemotherapy, anatomical site of the primary tumour or tumour grade; however, a trend in fewer cases of metastatic (AJCC Stage IV) cancer among Ala/Ala individuals was noted (see online supplementary table S3). Unstratified analysis using multifactorial logistic regression to control for race, grade and location of the primary tumour revealed a similar trend in fewer stage IV cancers diagnosed in $\mathrm{Ala} / \mathrm{Ala}$ individuals when analysed under the recessive model, which was significant when restricting the analysis to colon cancers (OR 0.30, $\mathrm{p}=0.017$ ) (table 1). Thus, ATG16L1 T300A was not significantly associated with tumour grade or location in this cohort. However, we observed fewer stage IV colon cancers in patients homozygous for T300A, suggesting that

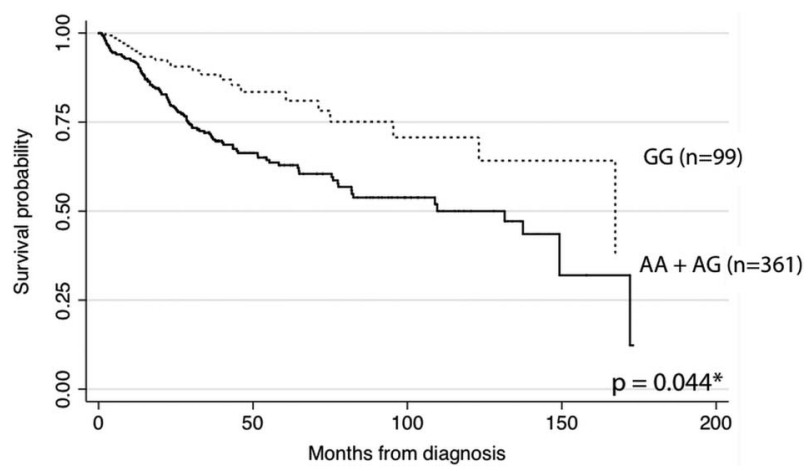

Figure 1 Adjusted Kaplan-Meier estimates of survival in colorectal cancer according to ATG16L1 genotype (recessive model). Data were centred on mean age for each genotype and adjusted for race and location of the primary tumour by Cox regression. * Stratified log-rank test and KM estimates were generated using STATA. differences in cancer progression may account for the association between ATG16L1 T300A and improved overall survival.

Previous studies describe a role for ATG16L1 in regulation of inflammation and antiviral immunity in the intestine ${ }^{25}$ and specifically in the suppression of a type I and II IFN-related gene signature. $^{26}$ Thus, we hypothesised that differences in diseasemodifying cytokines may underlie the association between the Ala/Ala genotype and prolonged survival. IFN-I is an important antiviral cytokine that initiates antitumor immunity, ${ }^{19} 20$ inhibits cancer cell growth ${ }^{12}$ and metastasis. ${ }^{16}$ Therefore, we hypothesised that ATG16L1 might regulate production of IFN-I in colon carcinoma cells.

\section{ATG16L1 T300A is associated with elevated MxA expression in human CRC}

The extent and significance of IFN-I gene expression in the pathophysiology of human CRC is not completely understood. The GTPase MxA is induced specifically in response to IFN-I and negatively regulated by ATG16L1 in murine colonic tissue. $^{26}$ To understand the significance of ATG16L1 genotype on MxA protein expression in human CRC, we tested whether the T300A polymorphism was associated with MxA expression in tumour sections from surgically resected formalin-fixed paraffin-embedded tissues. A blinded pathologist scored the extent of MxA expression within carcinoma cells on a scale of 1-4 (see online supplementary figure S2A). Tissue sections from $14 \mathrm{Thr} / \mathrm{Thr}$ and $12 \mathrm{Ala} / \mathrm{Ala}$ patients with confirmed adenocarcinoma were obtained and scores were averaged for each patient when multiple tumours were available. Expression of MxA in normal colonic epithelium was very rare (see online supplementary figure S2C). However, in contrast to adjacent non-dysplatic epithelium colon adenocarcinomas expressed varying degrees of MxA from none (MxA score 1) to $>30 \%$ of MxA-positive cancer epithelium (MxA score 4) (see online supplementary figure S2B). When stratified by genotype, we observed a significant association between ATG16L1 Ala/Ala patients and increased MxA scores $(p=0.048)$ (figure 2A, B). Thus, the T300A polymorphism in ATG16L1 is associated with elevated MxA expression in human CRC, raising the possibility that an elevated IFN-I response could underlie previous observations.

Table 1 Multifactorial regression of variables with respect to diagnosis with stage IV colon cancer

\begin{tabular}{|c|c|c|}
\hline Factor & OR $(95 \% \mathrm{Cl})$ & $\mathrm{p}$ Value \\
\hline \multicolumn{3}{|l|}{ ATG16L1 } \\
\hline$A A+A G$ & $1^{*}$ & \\
\hline \multicolumn{3}{|l|}{ Genotype } \\
\hline GG & 0.30 (0.11 to 0.81$)$ & 0.017 \\
\hline \multicolumn{3}{|l|}{ Age } \\
\hline$>60$ & $1^{*}$ & \\
\hline $60-70$ & $1.4(0.62$ to 3.1$)$ & 0.42 \\
\hline$>70$ & 0.99 (0.48 to 2.0$)$ & 0.98 \\
\hline \multicolumn{3}{|l|}{ Race } \\
\hline Caucasian & $1^{*}$ & \\
\hline African-American & $0.83(0.46$ to 1.5$)$ & 0.56 \\
\hline \multicolumn{3}{|l|}{ Grade } \\
\hline I & $1^{*}$ & \\
\hline ॥ & $2.0(0.77$ to 5.1$)$ & 0.15 \\
\hline III & $2.2(0.75$ to 6.4$)$ & 0.15 \\
\hline
\end{tabular}




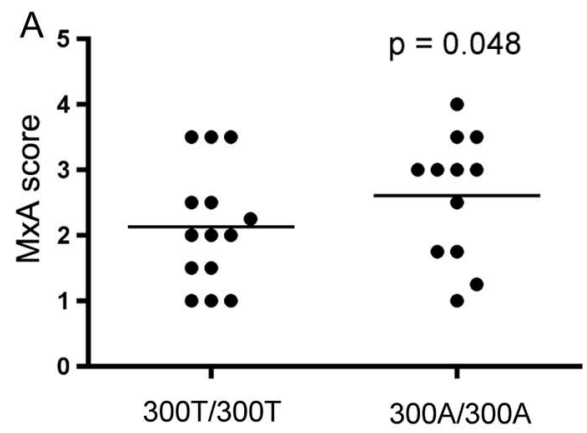

B

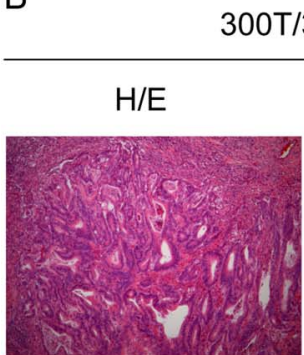

$300 \mathrm{~A} / 300 \mathrm{~A}$
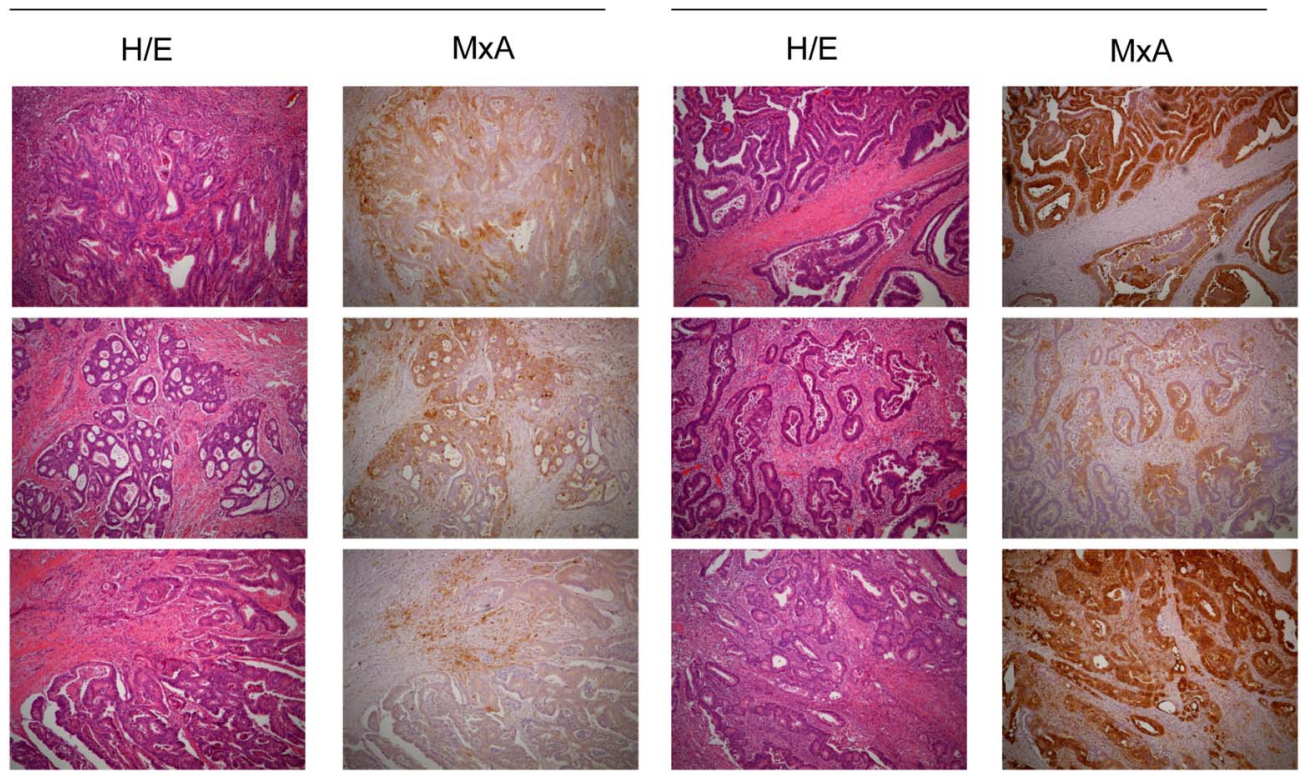

Figure 2 MxA expression in human adenocarcinoma with respect to ATG16L1 genotype. (A) MxA score graphed by ATG16L1 genotype in human colon adenocarcinomas. 300T/300T ( $N=14)$ and 300A/300A ( $N=12)$. Data analysed by Fisher's exact test. (B) immunohistochemical staining against MxA in human adenocarcinoma with respect to ATG16L1 genotype. Corresponding H/E shown in left panels (40x).

ATG16L1 T300A facilitates type I IFN production in CRC. To understand whether the T300A variant alters cancer cell physiology and production of IFN-I in vitro, we used homologous recombination to introduce this SNP into CRC cells. We also used this method to produce cells that are deficient $(-/-)$ in ATG16L1 expression and autophagy as we have previously described. $^{2}$ Cells deficient in ATG16L1 display defects in lipidation of LC3 and an accumulation of p62 indicative of a lack of substrate encapsulation and delivery to the lysosome (see online supplementary figure S3A, left panel). Conversely, bulk autophagy and lipidation of LC3 is not affected by introduction of the T300A variant in CRC cells (see online supplementary figure S3A, right panel). Thus, the T300A variant does not affect bulk autophagy in CRC cells, but may affect autophagy-independent functions.

Due to the negative regulation of MAVS and its cytosolic receptors by ATG5-ATG12 and the potential involvement of ATG16L1 in this complex, ${ }^{4} 27$ we performed mitochondrial fractionation to examine whether cytosolic receptors of MAVS, RIG-I and MDA5, were localised to the mitochondria. In ATG16L1 $1^{-/}$cells, we detected increased RIG-I and MDA5 within mitochondrial fractions, suggesting that ATG16L1 prevents translocation of these cytosolic receptors to the mitochondria (figure 3A). Deficiency in ATG16L1 did not impact mitochondrial mass in CRC cells (see online supplementary figures S3B, S3C) nor does it affect levels of other proteins implicated in regulation of MAVS including mitochondrial elongation factor Tu (TUFM) or ATG5-ATG12 within the crude mitochondrial fraction (NLRX1 was undetectable in these cells). In contrast, deficiency of ATG16L1 resulted in elevated expression of both cytosolic receptors of MAVS, RIG-I and MDA5, indicative of a type I IFN-stimulated $\operatorname{state}^{28}$ (figure $3 \mathrm{~A}$ ). Importantly, lentiviral transduction of full-length ATG16L1 into ATG16L1 ${ }^{-/-}$cells suppressed expression of RIG-I compared with empty vector controls (see online supplementary figure S4A). These results suggest that ATG16L1 controls expression of RIG-I and MDA5 and may prevent translocation of these proteins to the mitochondrial compartment where they can activate MAVS signalling.

In order to determine whether elevated expression of RIG-I and MDA5 was coincident with an IFN-I response, we examined expression and translocation of IRF 9 (ISGF3 $\gamma$ ) to the nuclear compartment by indirect immunofluorescence. ISGF3 $\gamma$ (p48/IRF9) is part of a trimeric complex that includes heterodimerised STAT1 and STAT2 after their phosphorylation by IFN-dependent Jak kinases. ${ }^{29}$ Nuclear translocation of ISGF3 $\gamma$ was observed in cells deficient in ATG16L1 as well as increased expression of ISGF3 $\gamma$ in whole-cell lysates (figure 3B, C), 

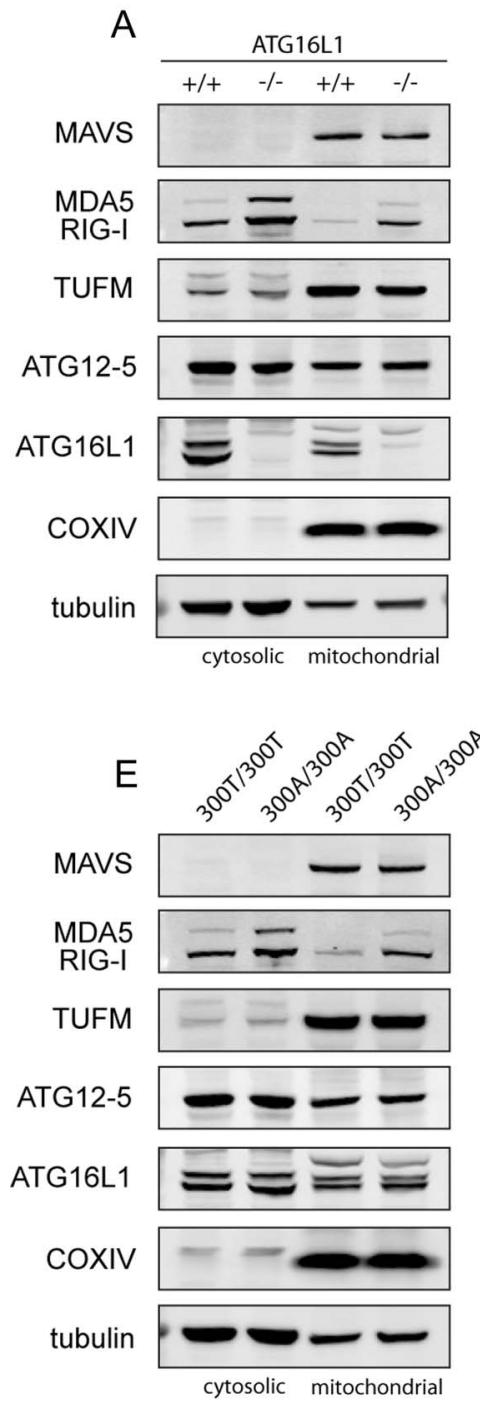

B

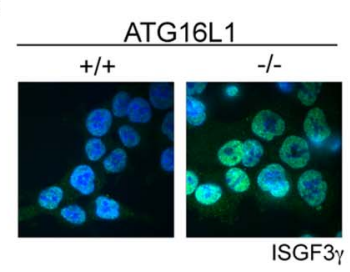

D

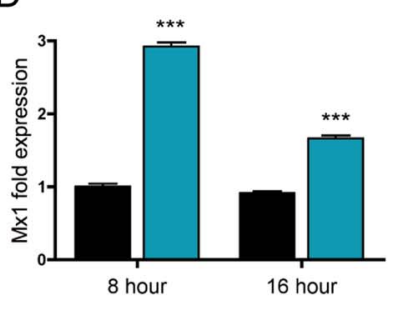

$\mathrm{F}$

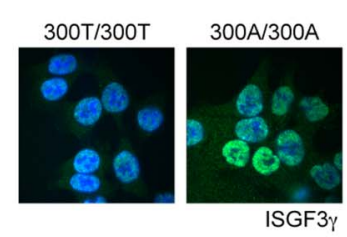

$\mathrm{H}$

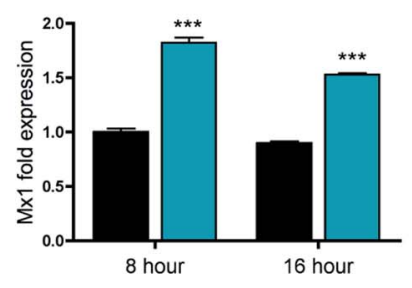

C
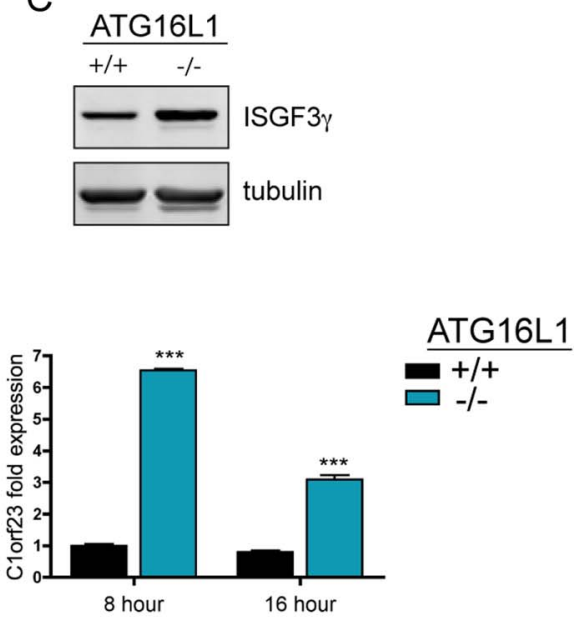

G

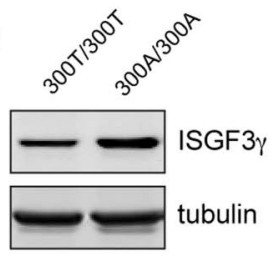

Figure 3 T300A impairs the ability of ATG16L1 to negatively regulate production of type I interferon in colorectal cancer cells. (A) Immunoblots of indicated proteins in crude mitochondrial lysates prepared from ATG16L1+/+ and ATG16L1 ${ }^{-1-}$ cells $(n=3)$. (B) Immunofluorescence staining for ISGF3 $\gamma$ (green) and Hoechst (blue) in ATG16L1+/+ and ATG16L1 ${ }^{-1-}$ cells. (C) Immunoblots of whole-cell lysates prepared from ATG16L1+/+ and ATG16L1 ${ }^{-I-}$ cells $(n=2)$. (D) Interferon (IFN) bioassay. Real-time PCR of type I interferon-sensitive genes in WISH cells stimulated with conditioned media collected from ATG16L1+/+ and ATG16L1 ${ }^{-I-}$ cells after indicated incubation period $(8$ or $16 \mathrm{~h})(\mathrm{n}=2)$. (E) Immunoblots of indicated proteins in crude mitochondrial lysates prepared from 300T/300T and 300A/300A cells $(n=3)$. (F) Immunofluorescence staining for ISGF3 $\gamma$ (green) and Hoechst (blue) in 300T/300T and 300A/300A cells $(n=2)$. (G) Immunoblots of whole-cell lysates prepared from 300T/300T and 300A/300A cells $(n=2)$. (H) IFN bioassay. Real-time PCR of type I interferon-sensitive genes in WISH cells stimulated with conditioned media collected from 300T/ $300 \mathrm{~T}$ and $300 \mathrm{~A} / 300 \mathrm{~A}$ cells after indicated incubation period $(8$ or $16 \mathrm{~h})(\mathrm{n}=2)$. Representative real-time PCR experiment performed in triplicate is shown $\left({ }^{* *} p<0.001\right.$, two-way ANOVA). ANOVA, analysis of variance; MAVS, mitochondrial antiviral signalling; MDA5, melanoma differentiation-associated protein 5; RIG-I, retinoic acid inducible gene I.

indicative of a response to IFN-I. ${ }^{29}$ In agreement with these results, induction of IFN-I-sensitive genes $\mathrm{Mx} 1(\mathrm{MxA})$ and C1orf23 was elevated in WISH cells after stimulation with supernatants collected from ATG16L1 $1^{-/}$compared with ATG16L1+/+ cells (figure 3D). Positive feedback of IFN-I is likely to contribute to elevated expression of ISGF3 $\gamma$ and RIG-I in ATG16 $\mathrm{L1}^{-/}$as these observations could be reproduced in ATG16L1 $+/+$ cells after stimulation with human recombinant IFN- $\beta$ for 1 (see online supplementary figure S5A) or $6 \mathrm{~h}$ (see online supplementary figure S5B). Thus, ATG16L1 suppresses production of IFN-I in CRC cells.

In order to examine whether the T300A variant of ATG16L1 affects the function of ATG16L1 to control production of IFN-I in CRC cells, we performed identical experiments comparing
ATG16L1 300A/300A cells to their 300T/300T counterparts. Similar to loss of ATG16L1, introduction of T300A into CRC cells resulted in increased production of IFN-I. Elevated expression and translocation of RIG-I and MDA5 to the mitochondrial fraction were evident in ATG16L1 T300A cells (figure 3E) as well as elevated expression and nuclear translocation of ISGF3 $\gamma$ (figure 3F, G). Finally, supernatants collected from cultured ATG16L1 T300A cells contained elevated IFN-I activity using the WISH bioassay (figure $3 \mathrm{H}$ ). Together, these data suggest that ATG16L1 functions to restrain production of IFN-I in CRC cells, and this negative regulation is reduced in cells with the T300A variant.

ATG16L1 T300A regulates production of MAVS-dependent type I IFN. Production of IFN-I can occur through two major 
signalling pathways involving TLR3-TRIF/TRAF3 or RIG-I/ MDA5-MAVS. ${ }^{30}$ These converge on common IRFs, which induce transcription of IFN- $\alpha / \beta$. In order to test whether ATG16L1 regulates MAVS-dependent production of IFN-I, we generated stably transduced cell lines expressing scrambled or one of two MAVS-targeted siRNAs and confirmed suppression of MAVS expression by immunoblotting whole cell lysates (figure 4A). Reduction of MAVS expression by $40-50 \%$ resulted in decreased expression of both RIG-I and ISGF3 $\gamma$ in ATG16L1 ${ }^{-/-}$and T300A cells, demonstrating that ATG16L1 may regulate IFN-I through the MAVS pathway.

To directly interrogate this pathway, we transfected CRC cells with the MDA5 ligand and dsRNA mimic, polyinosinic:polycytidylic acid (poly(I:C)). As opposed to naked poly(I:C), which stimulates primarily TLR3, transfection of poly(I:C)/Lyovec (Invivogen) is capable of stimulating cytosolic receptors leading to MAVS-dependent production of IFN-I. Compared with ATG16L1+/+ cells, ATG16L1 deficiency led to a dosedependent induction of IFN- $\beta$ mRNA in response to poly(I:C)/ Lyovec stimulation for $4 \mathrm{~h}$, suggesting that ATG16L1 participates in the response of CRC cells to poly(I:C) (figure 4B). Production of IFN- $\beta$ mRNA in response to poly(I:C)/Lyovec in ATG16L1 ${ }^{-/-}$cells was blunted by stable transduction of fulllength ATG16L1 (Figure S6A). Sensitivity of ATG16L1 ${ }^{-/-}$cells to poly(I:C)/Lyovec could be also be ablated by knock down of MAVS, confirming that the RIG-I/MDA5-MAVS pathway was primarily involved in this response (4C). Likewise, introduction of the T300A variant also sensitised CRC cells to poly(I:C)/ Lyovec (figure 4D) in a MAVS-dependent manner (figure 4E). Stimulation of genotyped CRC cell lines with poly(I:C)/Lyovec revealed induction of IFN- $\beta$ mRNA expression in ATG16 $1^{-/-}$ HCT116 cells and HT-29s, which are Ala/Ala, but not in parental HCT116 (Thr/Thr), SW480 (Thr/Thr) or DLD1 (Thr/Ala) cells (figure 4F). Taken together, these data suggest that ATG16L1 and the T300A variant regulates MAVS-dependent responses to poly(I:C)/Lyovec in CRC cells.

\section{DISCUSSION}

While the function of IFN-I in cancer has been appreciated for several decades, challenges related to delivery, efficacy and adverse events have made it difficult to target IFN in cancer therapy. ${ }^{11}{ }^{30} \alpha$-IFN acts as an adjuvant during antitumour vaccination, but use of IFN- $\alpha$ in conjunction with chemotherapies used to treat CRC has not been effective. ${ }^{31-33}$ Conversely, IFN- $\alpha$ has been used successfully in metastatic melanoma in high doses and in certain types of leukaemia. ${ }^{34}{ }^{35} \mathrm{It}$ is possible that adverse side effects may limit the potential of treatment with IFN- $\alpha$ to obtain an effective dose and durable response in CRC. Our results suggest that use of IFN-stimulating ligands in CRC therapy may be tailored by genotyping patients for ATG16L1 and potentially other variants that might impact IFN responses in CRC cells. Precisely how IFN-I promote survival in CRC is likely to be complex and involve cell autonomous and non-cell autonomous mechanisms. IFN-I production by a tumour cell would induce the recruitment and activation of immune cells, and the presence of such cells is one of the few variables known to improve the prognostic value of standard pathological assessments of CRC. ${ }^{36}$

Recent work is beginning to revive interest in targeting IFNs in other types of cancer. In human breast cancer, an elevated IFN-I expression signature is inversely related to bone metastasis recurrence. Cancer cells with reduced production of IFN-I due to lack of IRF7 had higher rates of bone metastasis in mice, suggesting that cancer cell-derived type I IFN might inhibit cancer progression. Oncolytic viruses are also known to preferentially target cancer cells and result in cell lysis due to defective antiviral pathways, whereas non-dysplastic epithelium are not permissive. ${ }^{37}$ The use of oncolytic viruses as immunoadjuvants in cancer treatment has passed safety and feasibility concerns in preclinical tests ${ }^{38-40}$ and has shown clinical promise in the treatment of human melanoma according to interim data. ${ }^{41}$ Activation of MAVS contributes to oncolytic virus-induced apoptosis in murine models of melanoma and orthotopic prostate cancer, identifying the importance of this pathway in the efficacy of oncolytic viruses. ${ }^{42}{ }^{43}$ Moreover, a subset of prostate cancer cell lines are resistant to oncolytic viruses in an IFN-I-dependent manner, suggesting that host genetics altering the IFN pathway could impact efficacy of this treatment. ${ }^{44} 45$ ATG16L1 T300A represents a potential host genetic factor that may increase sensitivity to therapies targeting the RIG-I-MAVS pathway or alter the response to viral infection in cancer cells. With more research studying T300A in various cancer types, genotyping patients for this variant could be important to predicting the efficacy of the aforementioned therapies.

Evidence pointing to a role for ATG16L1 in antiviral immunity is accumulating. ${ }^{4} 52746$ In the absence of IFN- $\alpha / \beta$, ATG16L1 is required for control of viral replication in response to IFN- $\gamma$ through unknown autophagy-independent mechanisms. ${ }^{5}$ ATG16L1-deficient mice succumb to Chikungunya virus infection, ${ }^{46}$ and colonic tissue lacking ATG16L1 expresses elevated type I and II IFN-related genes including $\mathrm{Mx},{ }^{26}$ in addition to a CD-like pathology after infection with murine norovirus. ${ }^{25}$ However, specific functions connecting ATG16L1 to regulation of antiviral immune mechanisms or viral replication have not yet been identified, nor is it understood whether the T300A variant impacts these functions. In a recent study, ATG16L1 was immunoprecipitated in complex with MAVS, suggesting that ATG16L1 and ATG5-ATG12 may act together to regulate production of type I IFN. ${ }^{27}$ ATG5-ATG12 directly inhibits activation of MAVS and production of type I IFN by interacting with MAVS and RIG-I and preventing association of their homotypic CARD domains in response to viral ligands. ${ }^{4}$ While ATG16L1 is a known partner of ATG5-ATG12, whether ATG16L1 is also required to regulate MAVS-dependent type I IFN is not known.

How ATG16L1 regulates production of IFN-I in CRC cells is currently under investigation, but previous studies point to both autophagy-dependent and autophagy-independent functions of autophagy proteins in this pathway. One possibility is that ATG16L1 (and T300A) regulates activation of MAVS signalling by altering mitochondrial function. Previous work has identified the importance of both mitochondrial membrane potential $\left(\Delta \Psi_{\mu}\right)$ and mitochondrial fusion in activation of MAVS. This is demonstrated by reduced MAVS activation after ectopic expression of the mitochondrial uncoupling protein, UCP-2, which dissipates $\Delta \Psi_{\mu}{ }^{47}$ In addition, cells lacking mitofusin-1 and mitofusin- 2 do not produce IFN- $\beta$ in response to Sendai virus infection, indicating that mitochondrial fusion could be required for organising a signalling platform on mitochondria. ${ }^{48}$ In contrast, an accumulation of depolarised reactive-oxygen-producing mitochondria in the context of ATG5 deficiency results in increased IFN- $\alpha$, which could be repressed by treatment with antioxidants. ${ }^{49}$ These studies contrast with direct inhibition of RIG-I-MAVS signalling by ATG5-ATG12 in an autophagy-independent manner. ${ }^{4}$ Moreover, in plasmocytoid dendritic cells, autophagy proteins have been shown to enhance rather than repress production of IFN-I due to their requirement in delivery of viral particles to the endosome where they 
A

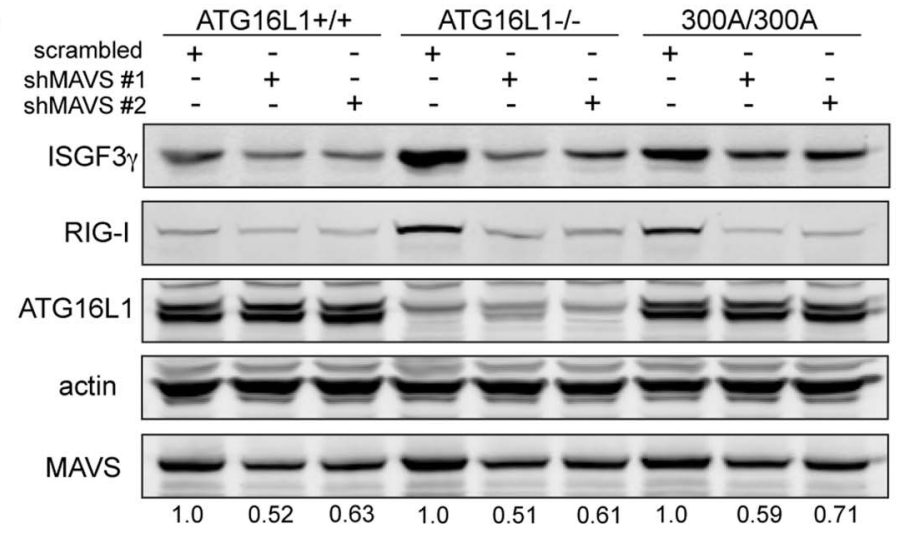

B

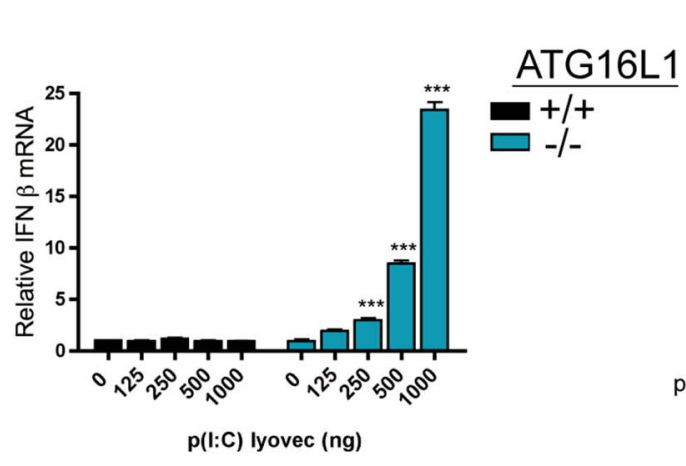

C

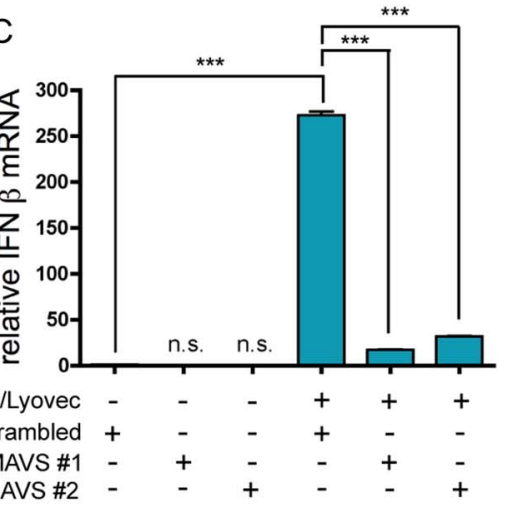

D

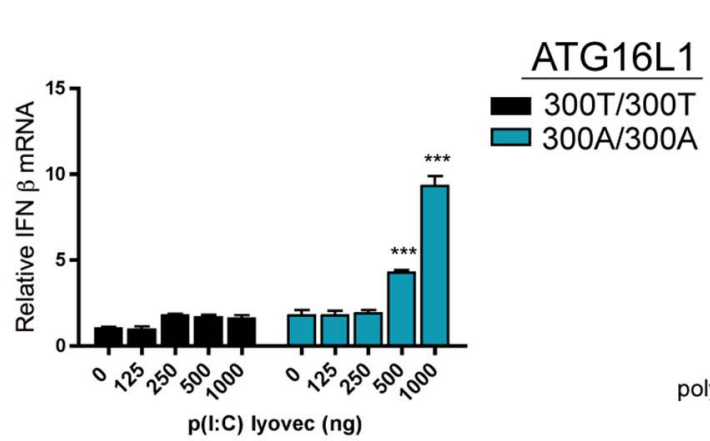

E

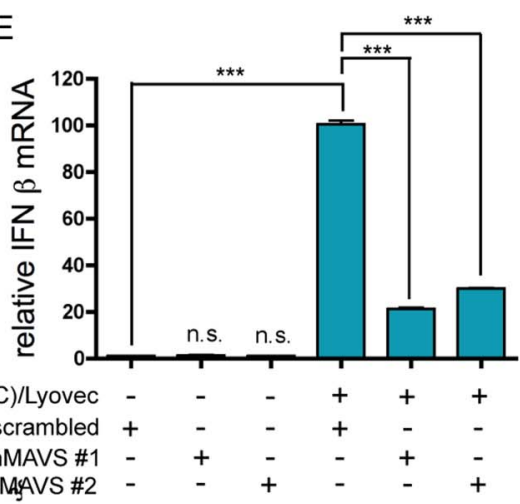

F

HCT116 Thr/Thr

HCT116 -/-

SW480 Thr/Thr

HT29 Ala/Ala

DLD1 Thr/Ala
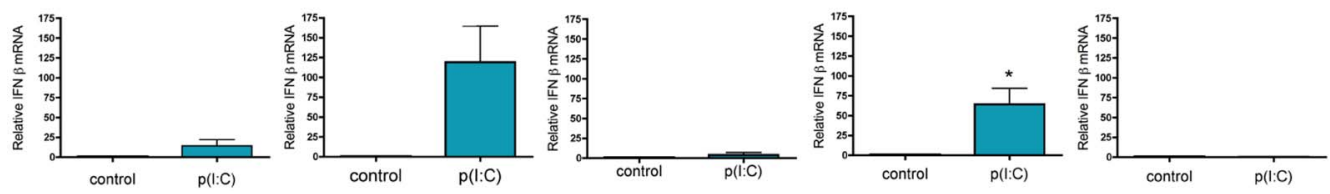

Figure 4 ATG16L1 regulates mitochondrial antiviral signalling (MAVS)-dependent type I interferon (IFN-I) production. (A) Immunoblotting of indicated proteins from whole-cells lysates prepared from ATG16L1 cell panel as indicated. Cells were lentivirally transduced with scrambled or one of two shRNAs targeting MAVS $(n=2)$. (B) Real-time PCR following stimulation of ATG16L1+l+ and ATG16L1 ${ }^{-l-}$ cells with poly(l:C) for $4 \mathrm{~h}(\mathrm{n}=2)$. (C) Real-time PCR following stimulation with $1000 \mathrm{ng} / \mathrm{mL}$ poly(l:C) for $4 \mathrm{~h}$ of ATG16L1 ${ }^{-1-}$ cells stably transduced with either scrambled or MAVS-targeted shRNAs ( $n=2$ ). (D) Real-time PCR following stimulation of ATG16L1 300T/300T and 300A/300A cells with poly(l:C) for $4 \mathrm{~h}$ ( $n=2$ ). (E) Real-time PCR following stimulation with $1000 \mathrm{ng} / \mathrm{mL}$ poly $(\mathrm{l}: \mathrm{C})$ for $4 \mathrm{~h}$ of ATG16L1 300A/300A cells stably transduced with either scrambled or MAVS-targeted shRNAs ( $\mathrm{n}=2$ ). (F) Real-time PCR following stimulation with $1000 \mathrm{ng} / \mathrm{mL}$ poly(l:C)/Lyovec for $4 \mathrm{~h}$ in common colorectal cancer cell lines. Representative real-time PCR experiment performed in triplicate is shown $\left({ }^{* *} p<0.001\right.$, two-way ANOVA). ANOVA, analysis of variance; RIG-I, retinoic acid inducible gene-I.

are recognised primarily by TLRs rather than RIG-I/MDA5. ${ }^{50}$ Thus, autophagy proteins have gene and cell-specific effects related to regulation of IFN-I. In CRC cells, we have not observed a significant accumulation of mitochondria upon ATG16L1. Based on these results, and functional bulk autophagy in T300A cells, we propose that ATG16L1 is likely to 
regulate IFN production through specific non-autophagy functions, which could include a requirement for ATG16L1 in inhibition of RIG-I/MAVS by ATG5-ATG12 or a specific role for ATG16L1 in mitochondrial polarisation or membrane dynamics.

We did not observe an association between ATG16L1 and age of diagnosis of CRC in this cohort; however, with increasing age and extended follow-up periods, competing comorbidities may influence survival analysis. Therefore, the lack of CRC-specific mortality data is a specific weakness of this study. Replication of these findings in a larger cohort with more complete survival data is underway.

Taken together, the data presented here support a broader role of ATG16L1 T300A in human pathophysiology. We found that ATG16L1 T300A is associated with improved survival and decreased metastasis in human CRC in conjunction with increased IFN-I activity in stage I adenocarcinoma. In addition to the known function of ATG5-ATG12 in regulation of MAVS, we have identified a requirement for ATG16L1 in negative regulation of MAVS-dependent IFN-I. In CRC cells, lack of ATG16L1 does not appear to alter ATG5-ATG12 protein expression in mitochondrial fractions, but we cannot rule out the possibility that ATG16L1 regulates IFN-I through its actions on ATG5-ATG12 or other indirect mechanisms.

Contributors Study concept and design: WAG, JSM, SFM, DH, MB, DLB; performed experiments: WAG, JSM, SFM, CRW, BES, AS, UD, DN; contributed key reagents and technical support: TN, JPL, MFL, SB, NE; wrote and edited manuscript: WAG, TBN, SSK, NE, MB, DLB.

Funding Grant support: Al083375-01; DK42086; Crohn's and Colitis Foundation of America 0-34493-1362; Broad medical Research Foundation IBD-0259 (to DLB) F32DK082104 (to JSM).

\section{Competing interests None.}

Patient consent Obtained.

Ethics approval Institutional Review Board of The University of Chicago.

Provenance and peer review Not commissioned; externally peer reviewed.

Open Access This is an Open Access article distributed in accordance with the Creative Commons Attribution Non Commercial (CC BY-NC 4.0) license, which permits others to distribute, remix, adapt, build upon this work non-commercially, and license their derivative works on different terms, provided the original work is properly cited and the use is non-commercial. See: http://creativecommons.org/ licenses/by-nc/4.0/

\section{REFERENCES}

1 Virgin HW, Levine B. Autophagy genes in immunity. Nat Immunol 2009:10:461-70.

2 Messer JS, Murphy SF, Logsdon MF, et al. The Crohn's disease-associated ATG16L1 variant and Salmonella invasion. BMJ Open 2013;3:pii: e002790.

3 Subramani S, Malhotra V. Non-autophagic roles of autophagy-related proteins. EMBO Rep 2013;14:143-51

4 Jounai N, Takeshita F, Kobiyama K, et al. The Atg5 Atg12 conjugate associates with innate antiviral immune responses. Proc Natl Acad Sci USA 2007:104:14050-5

5 Hwang S, Maloney NS, Bruinsma MW, et al. Nondegradative role of Atg5-Atg12/ Atg16L1 autophagy protein complex in antiviral activity of interferon gamma. Cell Host Microbe 2012;11:397-409.

6 Rioux JD, Xavier RJ, Taylor KD, et al. Genome-wide association study identifies new susceptibility loci for Crohn disease and implicates autophagy in disease pathogenesis. Nat Genet 2007;39:596-604.

7 Hampe J, Franke A, Rosenstiel P, et al. A genome-wide association scan of nonsynonymous SNPs identifies a susceptibility variant for Crohn disease in ATG16L1. Nat Genet 2007;39:207-11.

8 Kuballa P, Huett A, Rioux JD, et al. Impaired autophagy of an intracellular pathogen induced by a Crohn's disease associated ATG16L1 variant. PLOS ONE 2008;3: e3391.

9 Fujita N, Saitoh T, Kageyama S, et al. Differential involvement of Atg16L1 in Crohn disease and canonical autophagy: analysis of the organization of the Atg16L1 complex in fibroblasts. J Biol Chem 2009;284:32602-9.
10 Huijbers A, Plantinga TS, Joosten LA, et al. The effect of the ATG16L1 Thr300Ala polymorphism on susceptibility and outcome of patients with epithelial cell-derived thyroid carcinoma. Endocr Relat Cancer 2012;19:L15-18.

11 Fuertes MB, Woo SR, Burnett B, et al. Type I interferon response and innate immune sensing of cancer. Trends Immunol 2012;34:67-73.

12 Katayama T, Nakanishi K, Nishihara $\mathrm{H}$, et al. Type I interferon prolongs cell cycle progression via p21WAF1/CIP1 induction in human colon cancer cells. Int J Oncol 2007:31:613-20.

13 Kubler K, Gehrke N, Riemann S, et al. Targeted activation of RNA helicase retinoic acid-inducible gene-I induces proimmunogenic apoptosis of human ovarian cancer cells. Cancer Res 2010;70:5293-304.

14 Cao G, Su J, Lu W, et al. Adenovirus-mediated interferon-beta gene therapy suppresses growth and metastasis of human prostate cancer in nude mice. Cancer Gene Ther 2001;8:497-505.

15 Carretero R, Wang E, Rodriguez Al, et al. Regression of melanoma metastases after immunotherapy is associated with activation of antigen presentation and interferon-mediated rejection genes. Int J Cancer 2012;131:387-95.

16 Bidwell BN, Slaney CY, Withana NP, et al. Silencing of Irf7 pathways in breast cancer cells promotes bone metastasis through immune escape. Nat Med 2012;18:1224-31.

17 Burnette $B C$, Liang $H$, Lee $Y$, et al. The efficacy of radiotherapy relies upon induction of type $\mathrm{i}$ interferon-dependent innate and adaptive immunity. Cancer Res 2011;71:2488-96.

18 Kubler K, tho Pesch C, Gehrke N, et al. Immunogenic cell death of human ovarian cancer cells induced by cytosolic poly $(\mathrm{l}: \mathrm{C})$ leads to myeloid cell maturation and activates NK cells. Eur J Immunol 2011;41:3028-39.

19 Ogasawara K, Hida S, Azimi N, et al. Requirement for IRF-1 in the microenvironment supporting development of natural killer cells. Nature 1998;391:700-3

20 Swann JB, Hayakawa Y, Zerafa N, et al. Type I IFN contributes to NK cell homeostasis, activation, and antitumor function. J Immunol 2007;178:7540-9.

21 Hornung V, Ellegast J, Kim S, et al. 5' -Triphosphate RNA is the ligand for RIG-I. Science 2006;314:994-7.

22 Kawai T, Takahashi K, Sato S, et al. IPS-1, an adaptor triggering RIG-I- and Mda5-mediated type I interferon induction. Nat Immunol 2005;6:981-8.

23 Tang ED, Wang CY. MAVS self-association mediates antiviral innate immune signaling. J Virol 2009;83:3420-8.

24 Hua J, Kirou K, Lee C, et al. Functional assay of type I interferon in systemic lupus erythematosus plasma and association with anti-RNA binding protein autoantibodies. Arthritis Rheum 2006;54:1906-16.

25 Cadwell K, Patel KK, Maloney NS, et al. Virus-plus-susceptibility gene interaction determines Crohn's disease gene Atg16L1 phenotypes in intestine. Cell 2010;141:1135-45.

26 Marchiando AM, Ramanan D, Ding Y, et al. A deficiency in the autophagy gene Atg16L1 enhances resistance to enteric bacterial infection. Cell Host Microbe 2013;14:216-24.

27 Lei Y, Wen H, Yu Y, et al. The mitochondrial proteins NLRX1 and TUFM form a complex that regulates type I interferon and autophagy. Immunity 2012;36:933-46.

28 Hall JC, Rosen A. Type I interferons: crucial participants in disease amplification in autoimmunity. Nat Rev Rheumatol 2010;6:40-9.

29 Fu XY, Kessler DS, Veals SAJr, et al. ISGF3, the transcriptional activator induced by interferon alpha, consists of multiple interacting polypeptide chains. Proc Natl Acad Sci USA 1990:87:8555-9.

30 Trinchieri G. Type I interferon: friend or foe? J Exp Med 2010;207:2053-63.

31 Clark Pl, Slevin ML, Reznek RH, et al. Two randomised phase II trials of intermittent intravenous versus subcutaneous alpha- 2 interferon alone (trial 1) and in combination with 5 -fluorouracil (trial 2 ) in advanced colorectal cancer. Int $J$ Colorectal Dis 1987;2:26-9.

32 Gennatas C, Mouratidou D, Androulakis G, et al. Adjuvant systemic therapy protocol for Dukes' B2 and C resectable colon carcinoma. Tumori 2002;88:32-6.

33 Schippinger $W$, Jagoditsch $M$, Sorre $C$, et al. A prospective randomised trial to study the role of levamisole and interferon alfa in an adjuvant therapy with 5-FU for stage III colon cancer. Br J Cancer 2005;92:1655-62.

34 Tarhini AA, Gogas H, Kirkwood JM. IFN-alpha in the treatment of melanoma. J Immunol 2012;189:3789-93.

35 Quesada JR, Hersh EM, Manning J, et al. Treatment of hairy cell leukemia with recombinant alpha-interferon. Blood 1986;68:493-7.

36 Galon J, Mlecnik B, Bindea $G$, et al. Towards the introduction of the 'Immunoscore' in the classification of malignant tumours. J Pathol 2014;232:199-209.

37 Hammill AM, Conner J, Cripe TP. Oncolytic virotherapy reaches adolescence. Pediatr Blood Cancer 2010;55:1253-63.

38 Lun $X$, Ruan $Y$, Jayanthan $A$, et al. Double-deleted vaccinia virus in virotherapy for refractory and metastatic pediatric solid tumors. Mol Oncol 2013;7:944-54.

39 Heo J, Breitbach CJ, Moon A, et al. Sequential therapy with JX-594, a targeted oncolytic poxvirus, followed by sorafenib in hepatocellular carcinoma: preclinical and clinical demonstration of combination efficacy. Mol Ther 2011;19:1170-9.

40 Vasey PA, Shulman LN, Campos S, et al. Phase I trial of intraperitoneal injection of the E1B-55-kd-gene-deleted adenovirus ONYX-015 (dl1520) given on days 1 


\section{Colon}

through 5 every 3 weeks in patients with recurrent/refractory epithelial ovarian cancer. J Clin Oncol 2002;20:1562-9.

41 Sheridan C. Amgen announces oncolytic virus shrinks tumors. Nat Biotechnol 2013:31:471-2.

42 Matsushima-Miyagi T, Hatano K, Nomura M, et al. TRAIL and Noxa are selectively upregulated in prostate cancer cells downstream of the RIG-I/MAVS signaling pathway by nonreplicating Sendai virus particles. Clin Cancer Res 2012;18:6271-83.

43 Zhang Q, Xu X, Yuan Y, et al. IPS-1 plays a dual function to directly induce apoptosis in murine melanoma cells by inactivated Sendai virus. Int I Cancer 2014;134:224-34.

44 Moerdyk-Schauwecker M, Shah NR, Murphy AM, et al. Resistance of pancreatic cancer cells to oncolytic vesicular stomatitis virus: role of type I interferon signaling. Virology 2013;436:221-34.
45 Murphy AM, Besmer DM, Moerdyk-Schauwecker M, et al. Vesicular stomatitis virus as an oncolytic agent against pancreatic ductal adenocarcinoma. J Virol 2013;86:3073-87.

46 Joubert PE, Werneke SW, de la Calle $C$, et al. Chikungunya virus-induced autophagy delays caspase-dependent cell death. J Exp Med 2012;209:1029-47.

47 Koshiba T, Yasukawa K, Yanagi Y, et al. Mitochondrial membrane potential is required for MAVS-mediated antiviral signaling. Sci Signal 2011;4:77ra7.

48 Castanier C, Garcin D, Vazquez A, et al. Mitochondrial dynamics regulate the RIG-I-like receptor antiviral pathway. EMBO Rep 2010;11:133-8.

49 Tal MC, Sasai M, Lee HK, et al. Absence of autophagy results in reactive oxygen species-dependent amplification of RLR signaling. Proc Natl Acad Sci USA 2009;106:2770-5.

50 Lee HK, Lund JM, Ramanathan B, et al. Autophagy-dependent viral recognition by plasmacytoid dendritic cells. Science 2007;315:1398-401. 